\title{
Correction to: Effects of Different Combinations of Er:YAG Laser-Adhesives on Enamel Demineralization and Bracket Bond Strength. Photomed Laser Surg 2016;34:164-170; DOI: $10.1089 /$ pho.2015.4041
}

$\mathbf{T}$ HE ARTICLE ENTITLED, "Effects of Different Combinations of Er:YAG Laser-Adhesives on Enamel Demineralization and Bracket Bond Strength," published in Photomedicine and Laser Surgery 2016;34:164-170, requires correction.

In the abstract on page 164, there were numerical errors which were not discovered in the peer review process, as well as grammatical errors which were inadvertently bypassed during the production process. The confusion related to the number of teeth listed stems from the Materials and Methods section, which states that 108 teeth were used for SBS testing and 54 teeth were used for demineralization testing.

The revised abstract is listed below. The online version of the article has been corrected to reflect these changes.

\begin{abstract}
Objective: The purpose of this study was to investigate the demineralization around brackets and shear bond strength (SBS) of brackets bonded to Er:YAG laser irradiated enamel at different power settings with various adhesive systems combinations. Methods: A total of $\mathbf{1 0 8}$ premolar teeth were used for SBS testing and an additional 54 teeth were used to evaluate demineralization. Teeth were assigned into three groups according to the etching procedure, then each group divided into three subgroups based on the application of different adhesive systems. There were a total of nine groups as follows: Group 1: Acid + Transbond XT Primer; group 2: Er:YAG $(100 \mathrm{~mJ}, 10 \mathrm{~Hz})$ etching + Transbond XT Primer; group 3: Er:YAG $(200 \mathrm{~mJ}, 10 \mathrm{~Hz})$ etching + Transbond XT Primer; group 4: Transbond Plus self-etching primer (SEP); group 5: Er:YAG (100 mJ, $10 \mathrm{~Hz}$ ) etching + Transbond Plus SEP; group 6: Er:YAG $(200 \mathrm{~mJ}, 10 \mathrm{~Hz})$ etching + Transbond Plus SEP; group 7: Clearfil Protect Bond; group 8: Er:YAG $(100 \mathrm{~mJ}, 10 \mathrm{~Hz})$ etching + Clearfil Protect Bond; group 9: Er:YAG $(200 \mathrm{~mJ}, 10 \mathrm{~Hz})$ etching + Clearfil Protect Bond. Brackets were bonded with Transbond XT Adhesive Paste in all groups. Teeth to be evaluated for demineralization and SBS were exposed to $\mathrm{pH}$ and thermal cyclings, respectively. Then, demineralization samples were scanned with micro-CT to determine lesion depth values. For SBS test, a universal testing machine was used and adhesive remnant index was scored after debonding. Data were analyzed statistically. Results: No significant differences were found among the lesion depth values of the various groups, except for G7 and G8, in which the lowest values were recorded. The lowest SBS values were in G7, whereas the highest were in G9. The differences between the other groups were not significant. Conclusions: Er:YAG laser did not have a positive effect on prevention of enamel demineralization. When two step self-etch adhesive is preferred for bonding brackets, laser etching at $1 \mathrm{~W}(100 \mathrm{~mJ}, 10 \mathrm{~Hz})$ is suggested to improve SBS of brackets.
\end{abstract}

The authors regret this error. 